\title{
Adaptive Real-Time Energy Management Strategy for Plug-In Hybrid Electric Vehicle Based on Simplified-ECMS and a Novel Driving Pattern Recognition Method
}

\author{
Yuping Zeng $\mathbb{D},{ }^{1,2}$ Jing Sheng $\mathbb{D}^{1}{ }^{1}$ and Ming $\mathrm{Li} \mathbb{C}^{1}$ \\ ${ }^{1}$ Jiangxi Province Key Laboratory of Precision Drive \& Control, Nanchang Institute of Technology, Nanchang 330099, China \\ ${ }^{2}$ State Key Laboratory of Mechanical Transmissions, Chongqing University, Chongqing 400044, China \\ Correspondence should be addressed to Yuping Zeng; zengyp198410@163.com
}

Received 25 February 2018; Revised 6 August 2018; Accepted 27 September 2018; Published 14 October 2018

Academic Editor: Mauro Gaggero

Copyright (c) 2018 Yuping Zeng et al. This is an open access article distributed under the Creative Commons Attribution License, which permits unrestricted use, distribution, and reproduction in any medium, provided the original work is properly cited.

\begin{abstract}
This paper proposes an adaptive real-time energy management strategy for a parallel plug-in hybrid electric vehicle (PHEV). Three efforts have been made. First, a novel driving pattern recognition method based on statistical analysis has been proposed. This method classified driving cycles into three driving patterns: low speed cycle, middle speed cycle, and high speed cycle, and then carried statistical analysis on these three driving patterns to obtain rules; the types of real-time driving cycles can be identified according to these rules. Second, particle swarm optimization (PSO) algorithm is applied to optimize equivalent factor (EF) and then the EF MAPs, indexed vertically by battery's State of Charge (SOC) and horizontally by driving distance, under the above three driving cycles, are obtained. Finally, an adaptive real-time energy management strategy based on Simplified-ECMS and the novel driving pattern recognition method has been proposed. Simulation on a test driving cycle is performed. The simulation results show that the adaptive energy management strategy can decrease fuel consumption of PHEV by $17.63 \%$ under the testing driving cycle, compared to CD-CS-based strategy. The calculation time of the proposed adaptive strategy is obviously shorter than the time of ECMS-based strategy and close to the time of CD-CS-based strategy, which is a real-time control strategy.
\end{abstract}

\section{Introduction}

The energy crisis and environmental pollution problem have become a serious concern in the recent years [1]. Plug-in hybrid electric vehicles (PHEVs) assume an essential role in decreasing fuel consumption and pollutant emissions [2]. Indeed, the fuel economy and emissions performance of PHEVs is strongly influenced by many optimization tasks, that is, mechanical construction optimization [3], the powertrain parameters optimization [4], and the energy management strategy (EMS) optimization. The optimization of mechanical construction and the powertrain parameters is currently a well-proved technology. Nevertheless, the optimization-based energy management strategies (also called blended strategies) still have some implementation issues, such as high computation effort caused by algorithm complexity, robustness, and sensitivity to drive cycle information.
The optimization-based EMSs are classified into global optimization-based EMSs and instantaneous optimizationbased EMSs [5]. The global optimization-based EMSs mainly encompass dynamic programming- (DP-) based EMS [68], simulated annealing- (SA-) based EMS [9], and genetic algorithm- (GA-) based EMS [10]. DP-based EMS is able to compute global optimal solutions for PHEVs. Nonetheless, this EMS usually engenders "curse of dimensionality;" its computational burden increases exponentially with the number of states and control variables [11]. As a result, DPbased EMS is widely utilized in offline analysis to benchmark alternative EMSs [12], inspire RB strategies design [13], and serve as training data for machine learning algorithms [14]. SA and GA provide good performance even when dealing with complex problems [15]. They often combine with other algorithms to develop the EMS; that is, Chen et al. [9] derived an EMS based on combining SA with PMP for a PHEV; Chen et al. [10] combined GA with quadratic 
programming $(\mathrm{QP})$ to optimize the engine power and battery current in a power-split PHEV. SA-based EMS and GAbased EMS can obtain satisfactory results, but the calculation time is too long, making its online implementation difficult.

The instantaneous optimization-based-EMSs mainly encompass convex programming- (CP-) based-EMS [16-18], Pontryagin's minimum principle- (PMP-) based-EMS [1921], and equivalent consumption minimization- (ECMS-) based-EMS [22-24]. CP-based-EMS to PHEV has been reported in the recent literature. Zhang et al. [16] dealt with an analytical solution for the power management of a PHEV, where the vehicle model is simplified using quadratic equations. $\mathrm{Hu}$ et al. [17] designed two EMSs based on convex optimization in a series plug-in hybrid electric bus. Beck et al. [18] presented two approaches for an adaptive EMS with CP optimization, and both solutions were simulated; simulation results demonstrated that these solutions obtained near-optimal results with a significant decrease in computational time. CP-based-EMS simplifies the complexity of vehicle models and can attain near-optimal results in reduced time. Nevertheless, CP-based-EMS can only be applicable when the problem is strictly expressed in convex terms, which requires both cost function and inequality constraints expressed in convex form, and affine equality constraints $[25,26]$. Consequently, the application of such EMS is limited, owing to strong assumptions over the vehicle models. PMP-based-EMS transformed the global optimization problem to an instantaneous Hamiltonian optimization problem, which makes its real-time control possible. However, it is still a challenge for optimizing the Hamiltonian real-time due to the massive computational load required [27]. ECMS-based-EMS is a more promising option for energy management strategy of PHEV [28]. It was first introduced by Paganelli et al. in [22]. Sciarretta et al. [29] demonstrated that this EMS outperforms rule based EMS in a simulation environment. This EMS has also proved to be more computationally efficient than DP-based-EMS. However, the real-time application of ECMS-basedEMS requires further reduction of the calculation time [30].

To reduce the calculation time of the ECMS-based-EMS, we proposed a Simplified-ECMS-based EMS for a parallel plug-in hybrid electric vehicle in our previous article [31]. In that paper, the models of engine's fuel rate and battery's equivalent fuel consumption rate were approximately fitted by the piecewise function. Then, the total equivalent fuel rate was expressed by a convex piecewise function. According to the properties of convex functions, the ECMS problem was simplified to calculate and compare total equivalent fuel rate of only five candidates to identify the optimal torque distribution, instead of calculating and comparing the total equivalent fuel rate of huge candidates, who cover all over the control domain. In Simplified-ECMS-based EMS, the battery's electricity consumption is converted to an equivalent amount of fuel consumption using the equivalent factor (EF). EF is a key dynamic variable, which determines the practical implementation of Simplified-ECMS-based EMS. In our previous article [31], to get the suitable EF, we used extreme learning machine (ELM) for driving pattern recognition. ELM is a single layer feedforward neural network. As we all know, this neural network has high computational cost and is hard to be implemented to real-time control.

To solve the above problem and obtain an adaptive realtime energy management strategy for plug-in hybrid electric vehicle, a novel driving pattern recognition method with low computational cost was first proposed. This method classified driving cycles into three driving patterns: low speed cycle, middle speed cycle, and high speed cycle, carried statistical analysis on these three driving patterns to obtain rules, and identified the types of real-time driving cycles according to these obtained rules. Second, particle swarm optimization (PSO) algorithm is applied to optimize EF, and the EF MAPs under the above three driving cycles are obtained. Finally, an adaptive real-time energy management strategy based on Simplified-ECMS and the novel driving pattern recognition method was proposed. Simulation on a test driving cycle is performed to verify the proposed adaptive strategy.

This paper is outlined as follows. The structure and parameters of parallel CVT-based PHEV powertrain are described in Section 2, Simplified-ECMS-based EMS is introduced in Section 3, the adaptive real-time energy management strategy based on Simplified-ECMS and the novel driving pattern recognition method is presented in Section 4, and simulation results and discussions are provided in Section 5; this paper is ended with conclusion in Section 6.

\section{The Structure and Parameters of the Powertrain System}

This study focused on a single-shaft parallel CVT-based PHEV. Figure 1 shows the powertrain of this vehicle. The powertrain includes the internal combustion engine (ICE), the integrated starter and generator motor (ISG motor), the battery, the clutch, the continuously variable transmission (CVT), and final drive (FD). The vehicle runs in different operating modes by controlling the state of the engine and motor and the separation and combination of the clutch. According to the state of engine, the working mode of vehicle can be divided into two modes: engine on mode and engine off mode. During the engine on mode, the clutch is closed, the engine provides positive power, and the output power of the motor can be positive (driving), negative (generating), or zero (idle). During the engine off mode, only motor runs, and this mode can be subdivided into motor driving mode and braking mode. The basic parameters of the PHEV are shown in Table 1.

\section{Simplified-ECMS-Based EMS}

Simplified-ECMS-based EMS is derived from ECMS-based EMS. The detailed derivation process can be found in our previous article [31]. A brief description of the derivation process is given as follows:

Firstly, the engine's fuel rate and battery's equivalent fuel consumption rate are approximately fitted by the piecewise 


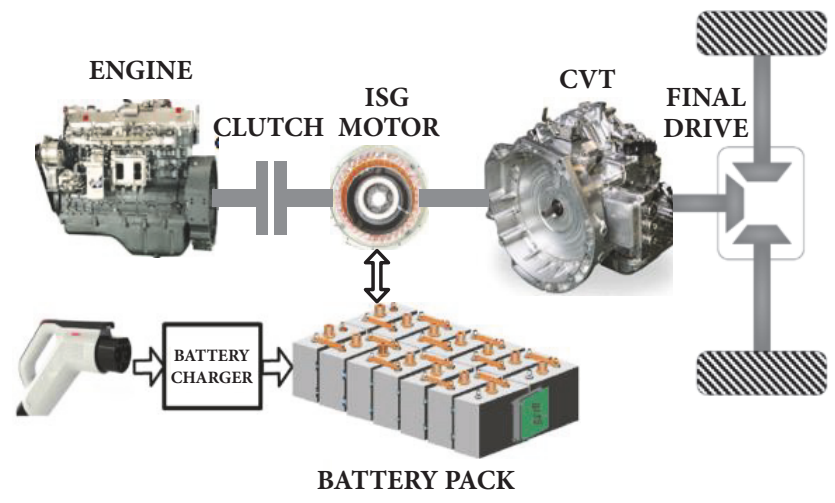

FIGURE 1: Parallel CVT-based PHEV powertrain system.

TABLE 1: Basic parameters of the PHEV.

\begin{tabular}{lcc}
\hline Components & Parameters & Value \\
\hline & Curb weight $/ \mathrm{kg}$ & 1395 \\
& Frontal area/m & 2.265 \\
& 0.301 & 0.307 \\
Basic parameters of the vehicle & Air drag coefficient & 0.0135 \\
& Wheel radius/m & 90 \\
& Wheel rolling resistance coefficient & 155 \\
Engine & Peak power/kW & 30 \\
& Maximum torque/(Nm) & 113 \\
ISG motor & Peak power/kW & 30 \\
& Maximum torque/(Nm) & 316 \\
Battery & Capacity/ Ah & 0.95 \\
& Rated voltage/V & 0.25 \\
\hline CVT & Initial SOC & $0.422-2.432$ \\
\hline FD & Minimum SOC & 5.24 \\
\hline
\end{tabular}

function. And the fitted result of the engine's fuel rate is expressed as follows [31]:

$$
\dot{m}_{i c e}= \begin{cases}\mathrm{c}_{10}+\mathrm{c}_{11} T_{m}+\mathrm{c}_{12} T_{m}{ }^{2} & \text { if } T_{\text {req }}-T_{\text {opt }} \leq T_{m} \leq \min \left(T_{\text {req }}-T_{\text {e min }}, T_{\max }\right) \\ c_{20}+c_{21} T_{m}+c_{22} T_{m}{ }^{2} & \text { if } \max \left(T_{r e q}-T_{\text {e max }}, T_{\text {min }}\right) \leq T_{m} \leq T_{\text {req }}-T_{o p t} \\ 0 & \text { if } T_{m}=T_{\text {req }}\end{cases}
$$

where $\dot{m}_{i c e}$ is the engine's fuel rate, $\mathrm{c}_{1 i}, \mathrm{c}_{2 i}(i=0,1,2)$ are constants, $T_{r e q}$ is the required torque needed to be distributed between engine and motor, $T_{e}$ is the output torque of engine, $T_{m}$ is the output torque of motor, $T_{o p t}$ is the engine's torque corresponding to the best efficiency point at a certain engine speed, $T_{\mathrm{e} \text { min }}$ is the engine's minimum output torque, $T_{\mathrm{e} \text { max }}$ is the engine's maximum output torque, and $T_{\min }$ and $T_{\max }$ are the minimum and maximum output torque of motor, respectively.

The fitted result of the battery's equivalent fuel consumption rate is expressed as follows [31]:

$$
\dot{m}_{b a t}= \begin{cases}\lambda C_{b 10}+\lambda C_{b 11} T_{m}+\lambda C_{b 12} T_{m}^{2} & \text { if } T_{m}<0 \\ \lambda C_{b 20}+\lambda C_{b 21} T_{m}+\lambda C_{b 22} T_{m}^{2} & \text { if } T_{m} \geq 0\end{cases}
$$

where $\dot{m}_{b a t}$ is the battery's equivalent fuel consumption rate, $C_{b 1 i}$ and $C_{b 2 i}(i=0,1,2)$ are constants, and $\lambda$ is the equivalent factor $(\mathrm{EF})$.

Then, the total equivalent fuel rate can be expressed by a convex piecewise function [31]:

(1) If $T_{\text {req }}-T_{o p t}<0$, then 


$$
\dot{m}_{e q}= \begin{cases}c_{30}+c_{31} T_{m}+c_{32} T_{m}{ }^{2} & \text { if } \max \left(T_{r e q}-T_{\mathrm{e} \max }, T_{\min }\right) \leq T_{m}<T_{r e q}-T_{o p t} \\ c_{40}+c_{41} T_{m}+c_{42} T_{m}{ }^{2} & \text { if } T_{r e q}-T_{o p t} \leq T_{m}<0 \\ c_{50}+c_{51} T_{m}+c_{52} T_{m}{ }^{2} & \text { if } 0 \leq T_{m} \leq \min \left(T_{r e q}-T_{\text {e min }}, T_{\max }\right) \\ \lambda \cdot \mathrm{L}= \begin{cases}\lambda C_{b 10}+\lambda C_{b 11} T_{m}+\lambda C_{b 12} T_{m}^{2} & \text { if } T_{m}=T_{r e q}<0 \\ \lambda C_{b 20}+\lambda C_{b 21} T_{m}+\lambda C_{b 22} T_{m}^{2} & \text { if } T_{m}=T_{r e q} \geq 0\end{cases} \end{cases}
$$

where $c_{30}=c_{20}+\lambda C_{b 10}, c_{31}=c_{21}+\lambda C_{b 11}, c_{32}=c_{22}+$ $+\lambda C_{b 12}, c_{40}=c_{10}+\lambda C_{b 10}, c_{41}=c_{11}+\lambda C_{b 11}, c_{42}=$ $c_{12}+\lambda C_{b 12}, c_{50}=c_{10}+\lambda C_{b 20}, c_{51}=c_{11}+\lambda C_{b 21}, c_{52}=c_{12}+$ $\lambda C_{b 22}$.

(2) If $T_{r e q}-T_{o p t} \geq 0$, then

$$
\dot{m}_{e q}=\left\{\begin{array}{l}
\mathrm{c}_{30}+\mathrm{c}_{31} T_{m}+\mathrm{c}_{32} T_{m}^{2} \\
\mathrm{c}_{60}+\mathrm{c}_{61} T_{m}+\mathrm{c}_{62} T_{m}{ }^{2} \\
\mathrm{c}_{50}+\mathrm{c}_{51} T_{m}+\mathrm{c}_{52} T_{m}^{2} \\
\lambda \cdot \mathrm{L}= \begin{cases}\lambda C_{b 10}+\lambda C_{b 11} T_{m}+\lambda C_{b 12} T_{m}^{2} & \text { if } T_{m}=T_{r e q}<0 \\
\lambda C_{b 20}+\lambda C_{b 21} T_{m}+\lambda C_{b 22} T_{m}^{2} & \text { if } T_{m}=T_{r e q} \geq 0\end{cases}
\end{array}\right.
$$

where $\mathrm{c}_{60}=\mathrm{c}_{20}+\lambda C_{b 20}, \mathrm{c}_{61}=\mathrm{c}_{21}+\lambda C_{b 21}, \mathrm{c}_{62}=\mathrm{c}_{22}+\lambda C_{b 22}$.

According to Eqs. (3) and (4), the total equivalent fuel rate is a piecewise function, which is composed of four continuous convex quadratic functions. Based on the property of convex function, the total equivalent fuel rate is also a convex function. Therefore, the minimum value of the total equivalent fuel rate can only be obtained at points of demarcation of the convex piecewise function [31]. As shown in Eqs. (3) and (4), the demarcation points of the piecewise function are $T_{m}=\max \left(T_{\text {req }}-T_{\mathrm{e} \max }, T_{\min }\right), T_{m}=T_{\text {req }}-T_{o p t}, T_{m}=0$, $T_{m}=\min \left(T_{\text {req }}-T_{\text {e min }}, T_{\max }\right)$, and $T_{m}=T_{\text {req }}$. Thus, the optimal control variable can only be obtained from above five points. The optimal solution can be determined by comparing the values of the total equivalent fuel rate of the five points.

Consequently, the concept of the Simplified-ECMS-based EMS is achieving the optimal solution by calculating and comparing the total equivalent fuel rate of the above five points for each time instant, instead of calculating and comparing the total equivalent fuel rate of huge candidates, who cover all over the control domain [31]. Therefore, the Simplified-ECMS-based EMS can greatly reduce the amount of calculation and shorten the time of calculation. The procedure flow chart of the Simplified-ECMS-based EMS is shown in Figure 2. $T_{m 1}, T_{m 2}, T_{m 3}, T_{m 4}$, and $T_{m 5}$ are the five demarcation points, $\dot{m}_{e q}\left(T_{m 1}\right), \dot{m}_{e q}\left(T_{m 2}\right), \dot{m}_{e q}\left(T_{m 3}\right)$, $\dot{m}_{e q}\left(T_{m 4}\right)$, and $\dot{m}_{e q}\left(T_{m 5}\right)$ are the corresponding total equivalent fuel rates of above five points. The operating principle of this EMS is as follows:

(1) Calculate the required torque $T_{\text {req }}$ according to the driver's pedal. And, if $T_{\text {req }}<0$, then the vehicle runs in braking energy recovery mode.
(2) If $T_{r e q} \geq 0$, then calculate five demarcation points.

(3) Obtain the equivalent factor (EF) by a certain algorithm and then calculate the total equivalent fuel rate of above five points according to Eqs. (3) and (4).

(4) Obtain the optimal control variable after comparing the total equivalent fuel rates of above five points, and output the optimal torque distribution to power system.

\section{The Adaptive Real-Time Energy Management Strategy Based on Simplified- ECMS and the Novel Driving Pattern Recognition Method}

The detailed development process of the adaptive realtime energy management for the plug-in HEV based on simplified-ECMS and the novel driving pattern recognition method is illustrated in Figure 3. It mainly consists of two parts, that is, the offline part and the online part. The offline part has three modules, three typical driving cycles, probability density function of two driving features under three typical driving cycles, and global offline optimizer based on PSO algorithm, while the online part mainly includes four modules, driving pattern recognition, look-up MAPs of EF, simplified-ECMS-based EMS, and powertrain system.

The logic flow is also shown in Figure 3. To clearly show the proposed strategy, the detailed procedure is shown in Table 2. There are a total of 8 steps.

In step 1, three typical driving cycles (low speed cycle, middle speed cycle, and high speed cycle) are constructed after driving data gathering, driving features selection, and driving segments clustering. The profiles of three typical driving cycles are shown in Figure 4. 


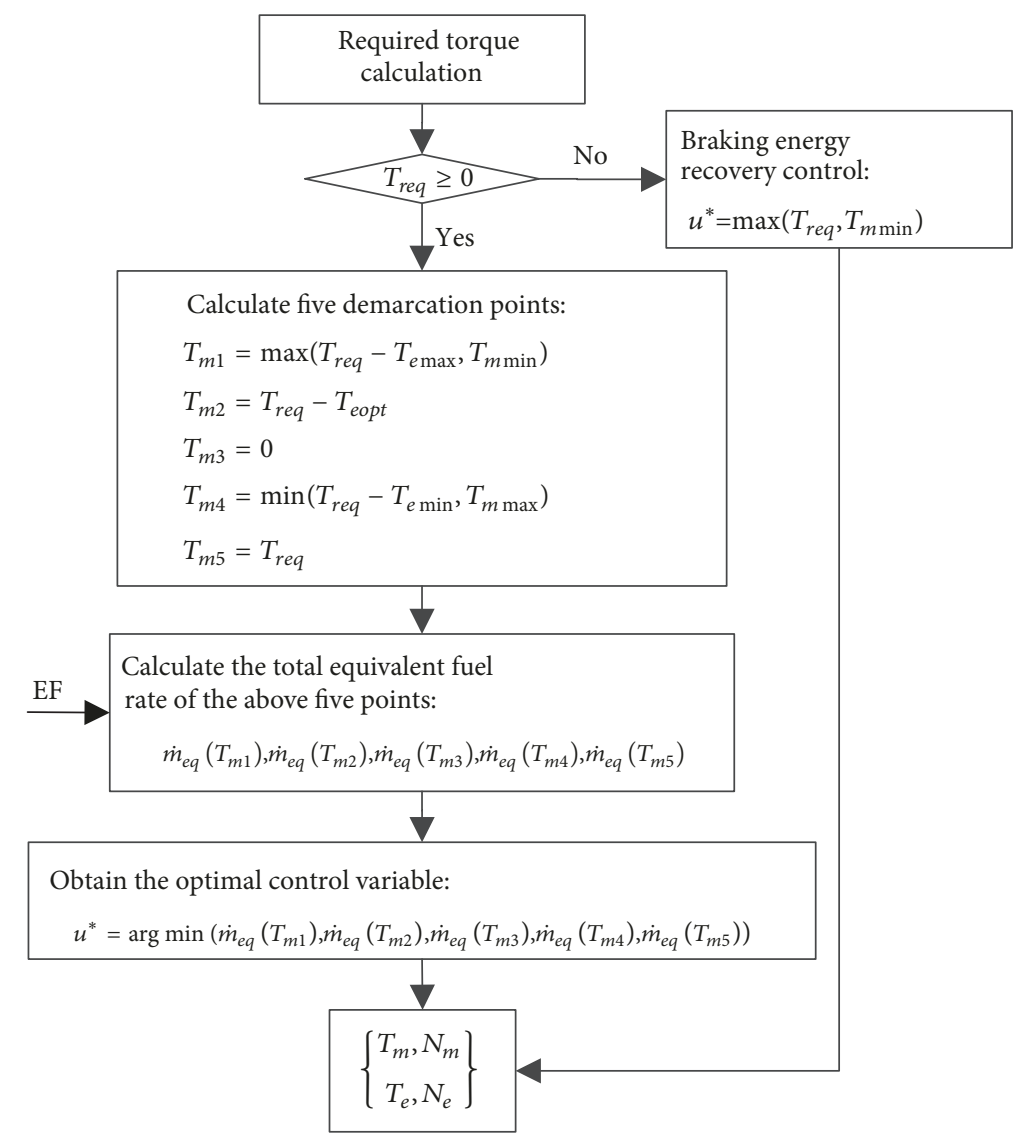

FIgURE 2: The procedure flow chart of the Simplified-ECMS-based EMS.

TABLE 2: Detailed procedure of the proposed energy management strategy.

(1) Three typical driving cycles are constructed after driving data gathering, driving features selection, and driving segments clustering.

(2) Two driving features for driving pattern recognition are determined, and the probability density functions of these two driving features under the above typical driving cycles are obtained by statistical analysis.

Offline

(3) Rules of driving cycle recognition are extracted according to the above two probability density functions.

(4) Particle swarm optimization (PSO) algorithm is applied to optimize equivalent factor (EF), and the MAPs of this factor under different typical driving cycles, driving distance, and SOC are obtained.

(5) The real-time driving pattern is identified according to the driving cycle recognition rules, and output the type of drive cycle.

(6) The driving distance is got by navigation system and vehicle's velocity.

Online

(7) Based on the aforementioned work, the EF can be obtained by looking up EF Maps through the type of driving cycle, driving distance, and SOC.

(8) Simplified-ECMS-based strategy is employed to solve the energy distribution optimization problem, and the optimal torque distribution between engine and motor can be obtained.

In step 2 and step 3, mean velocity $\left(V_{\text {mean }}\right)$ and cruise percentage $\left(t_{\text {cruise }} / t_{\text {total }} \times 100\right)$ are chosen for driving pattern recognition, and the probability density functions of these two driving features under the above typical driving cycles are obtained by statistical analysis. These probability density functions are shown in Figure 5. $f_{\text {low }}, f_{\text {mid }}$, and $f_{\text {hig }}$ are the probability of low speed cycle, middle speed cycle, and high speed cycle, respectively. $V_{\text {mean_A }}$ is the mean velocity corresponding to the A point, $V_{\text {mean_B }}$ is the mean velocity corresponding to the B point, $P_{c r u_{-} C}$ is the cruise percentage corresponding to the $\mathrm{C}$ point, and $P_{c r u} D$ is the cruise percentage corresponding to the $\mathrm{D}$ point. In this study, the 


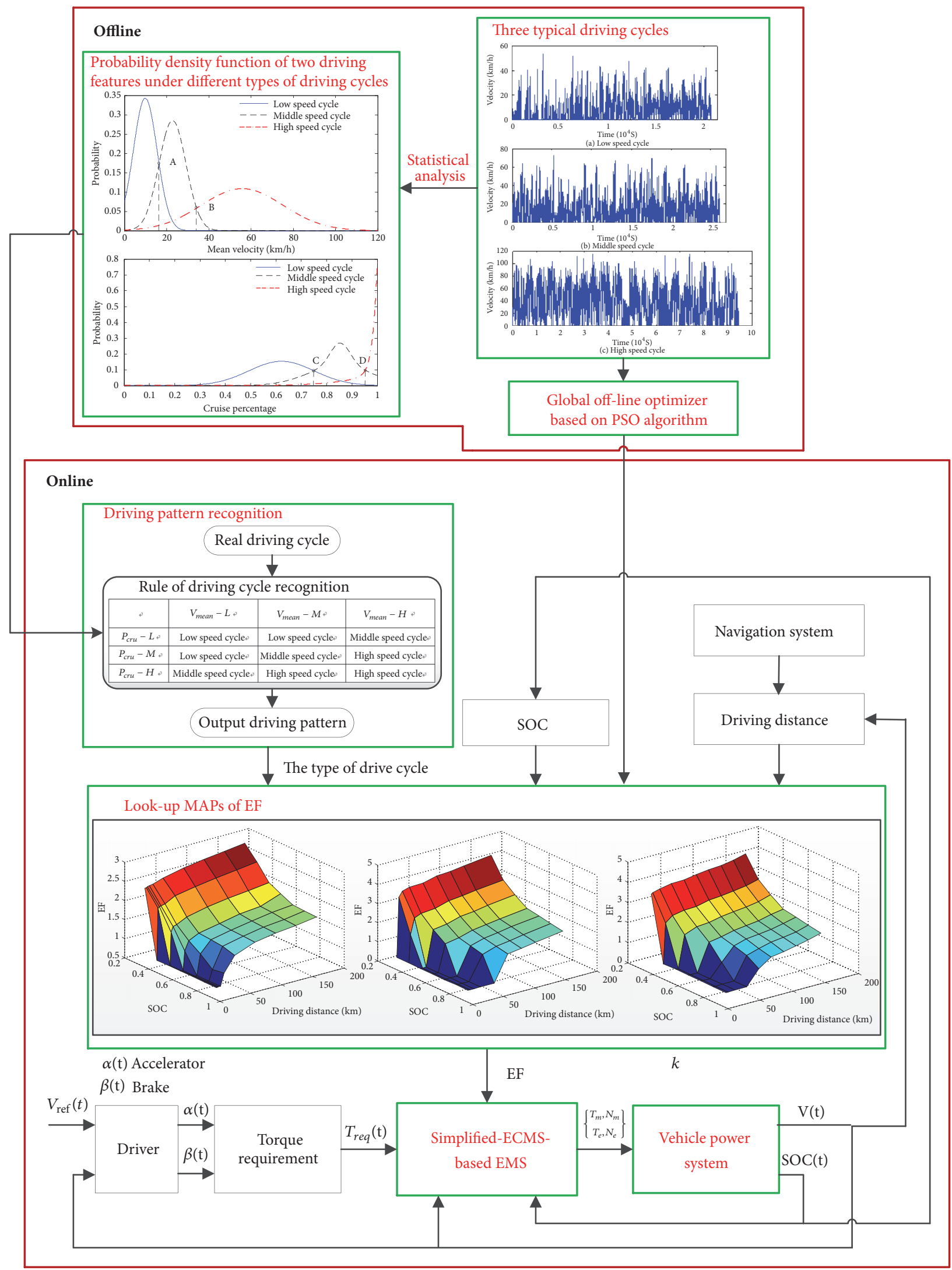

FIGURE 3: Control scheme of the adaptive energy management strategy for the plug-in HEV. 


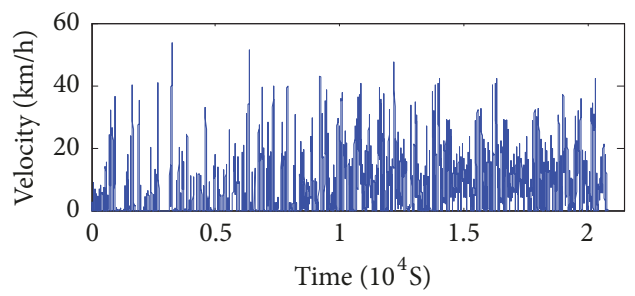

(a) Low speed cycle

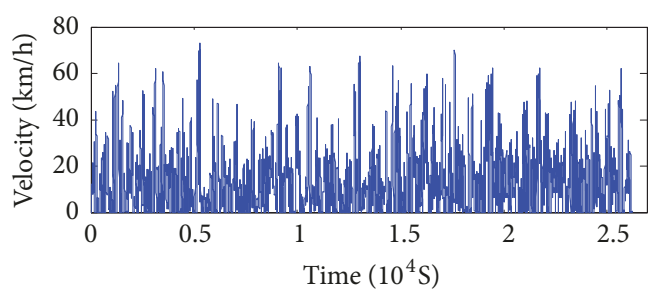

(b) Middle speed cycle

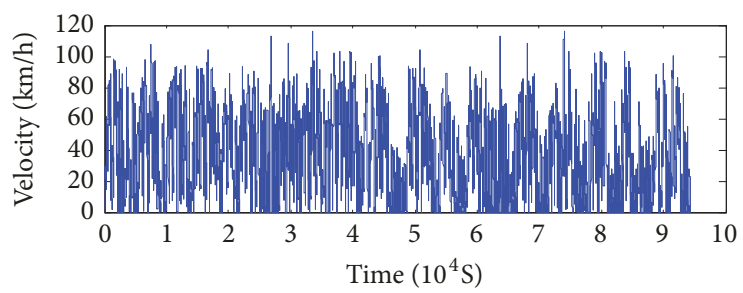

(c) High speed cycle

FIGURE 4: Profiles of three typical driving cycles.

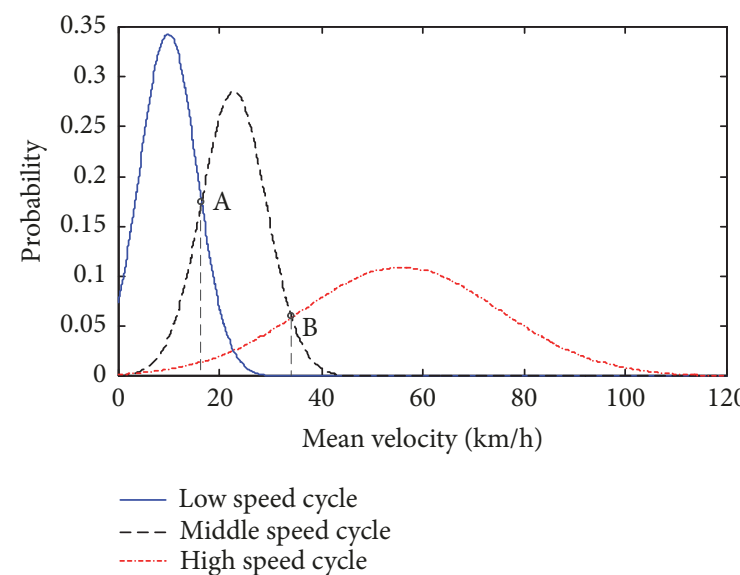

(a) Probability density function of mean velocity under different types of driving cycles

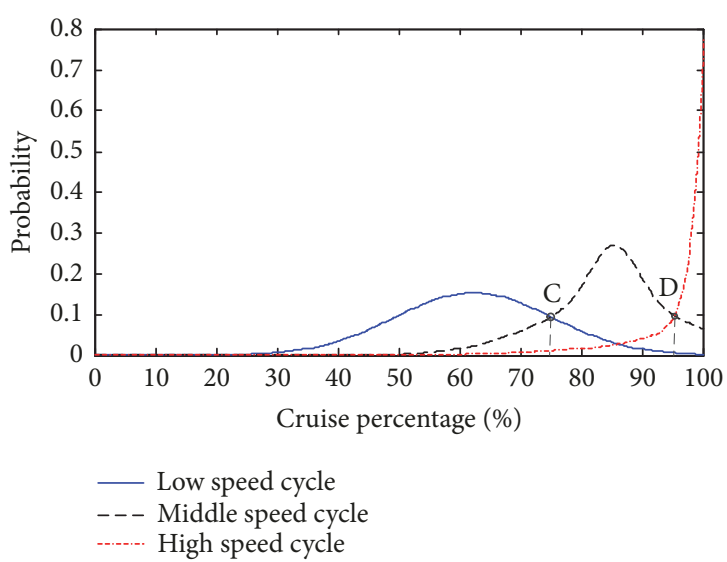

(b) Probability density function of cruise percentage under different types of driving cycles

FIGURE 5: The probability density functions of two driving features under different driving cycles.

previous $100 \mathrm{~s}$ duration of historical information has been determined to identify a real-time driving pattern. $V_{\text {mean }}(t)$ is the mean velocity of the past $100 \mathrm{~s} . P_{c r u}(t)$ is the cruise percentage of the past $100 \mathrm{~s}$. Then, we can obtain the following deduction according to Figure 5.

(1) If $V_{\text {mean }}(t) \leq V_{\text {mean_A }}$, then $f_{\text {low }} \geq\left(f_{\text {mid }}, f_{\text {hig }}\right)$, and the current driving cycle is inclined to low speed cycle, and this state is expressed as $V_{\text {mean }}-L$.

(2) If $V_{\text {mean_A }}<V_{\text {mean }}(t)<V_{\text {mean_B }}$, then $f_{\text {mid }}>$ $\left(f_{\text {low }}, f_{\text {hig }}\right)$, and the current driving cycle is inclined to middle speed cycle, and this state is expressed as $V_{\text {mean }}-M$.

(3) If $V_{\text {mean }}(t) \geq V_{\text {mean_B }}$, then $f_{\text {hig }} \geq\left(f_{\text {low }}, f_{\text {mid }}\right)$, and the current driving cycle is inclined to high speed cycle, and this state is expressed as $V_{\text {mean }}-H$.
(4) If $P_{\text {cru }}(t) \leq P_{\text {cru_C }}$, then $f_{\text {low }} \geq\left(f_{\text {mid }}, f_{\text {hig }}\right)$, and the current driving cycle is inclined to low speed cycle, and this state is expressed as $P_{c r u}-L$.

(5) If $P_{\text {cru_C }}<P_{\text {cru }}(t)<P_{\text {cru_D }}$, then $f_{\text {mid }}>\left(f_{\text {low }}, f_{\text {hig }}\right)$, and the current driving cycle is inclined to middle speed cycle, and this state is expressed as $P_{c r u}-M$.

(6) If $P_{\text {cru }}(t) \geq P_{\text {cru-D }}$, then $f_{\text {hig }} \geq\left(f_{\text {low }}, f_{\text {mid }}\right)$, and the current driving cycle is inclined to high speed cycle, and this state is expressed as $P_{c r u}-H$.

Based on the above deduction, the rules for driving cycle recognition are determined, and these rules are shown in Table 3. In this table, Low stands for the low speed cycle, Middle stands for the middle speed cycle, and High stands for the high speed cycle. 


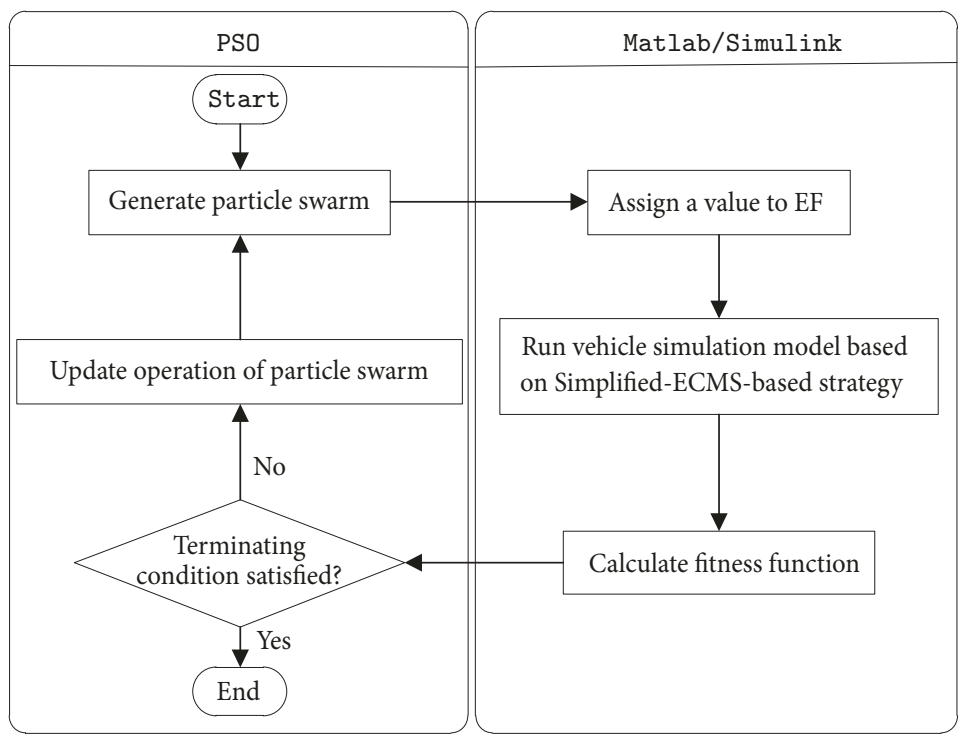

FIgURE 6: The flow chart of EF optimization.

TABLE 3: Rules for the driving pattern recognition.

\begin{tabular}{lccc}
\hline & $V_{\text {mean }}-L$ & $V_{\text {mean }}-M$ & $V_{\text {mean }}-M$ \\
\hline$P_{\text {cru }}-L$ & Low & Low & Middle \\
$P_{c r u}-M$ & Low & Middle & High \\
$P_{c r u}-H$ & Middle & High & High \\
\hline
\end{tabular}

In Step 4, particle swarm optimization (PSO) algorithm is applied to optimize equivalent factor (EF) of the simplifiedECMS-based EMS under each typical driving cycle, driving distance, and SOC. The fitness function is expressed as

$$
\begin{aligned}
F & =\min _{\{k\}} \int_{0}^{t} \dot{m}_{i c e}(\mathrm{t}) \mathrm{d} t \\
S O C_{\text {min }} & \leq S O C(\mathrm{t}) \leq S O C_{\text {max }} \\
T_{\text {emin }}(\mathrm{t}) & \leq T_{e}(\mathrm{t}) \leq T_{\text {emax }}(\mathrm{t}) \\
T_{\text {mmin }}(\mathrm{t}) & \leq T_{m}(\mathrm{t}) \leq T_{\text {mmax }}(\mathrm{t})
\end{aligned}
$$

The flow chart of EF optimization is shown in Figure 6, the simulation is performed under high speed cycle, the driving distance is $66.02 \mathrm{~km}$, and the SOC is 0.95 . These parameters of PSO are set as follows: the size of particle swarm is 100, the maximum number of iterations is 100 , the initial inertia weight is 0.9 , and the final inertia weight is 0.4 .

The optimization result is shown in Figure 7, the optimal value of EF is 2.277, and the optimal fuel consumption is 2.675L.

$\mathrm{EF}$ is mainly affected by driving distance and SOC in certain driving cycle, the above EF optimization is based on given driving distance and SOC; next, under high speed cycle, the EF optimization is implemented one by one in different driving distances and SOC through offline optimization, and the MAP figure of EF under high speed cycle, as shown in Figure 8 , is obtained. In the same way, the MAPs figure of

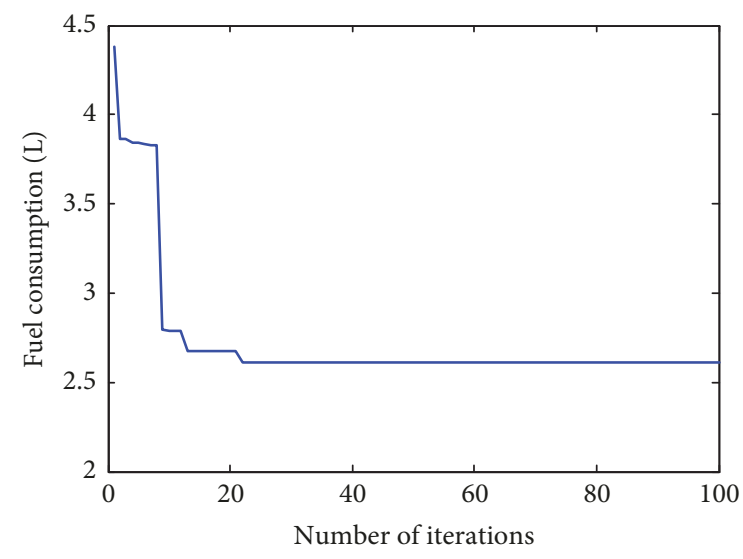

FIgURE 7: The optimization result.

EF under middle speed cycle and low speed cycle are also obtained, and they are shown in Figures 9 and 10, respectively. As shown in Figures 8, 9, and 10, when the driving distance is less than the pure electric mileage, EF is a constant, and its value is minimum; the reason is that electrical energy is enough in this condition; a small EF makes driving using battery power as much as possible; when the driving distance is larger than the pure electric mileage, EF increases with the increasing driving distance in certain SOC, which can blend electric energy with the trip distance adding. EF reduces with the increasing SOC in certain driving distance; then, the opportunity of motor participation increases in this way.

In Step 5, the real-time driving pattern is identified according to the driving cycle recognition rules. In this step, the time window of data sampling is set to $100 \mathrm{~s}$. Once the vehicle is running, the vehicle controller will monitor the vehicle speed. When the operating time exceeds $100 \mathrm{~s}$, the driving cycle recognition module will calculate mean velocity and cruise percentage for the previous driving block, and 


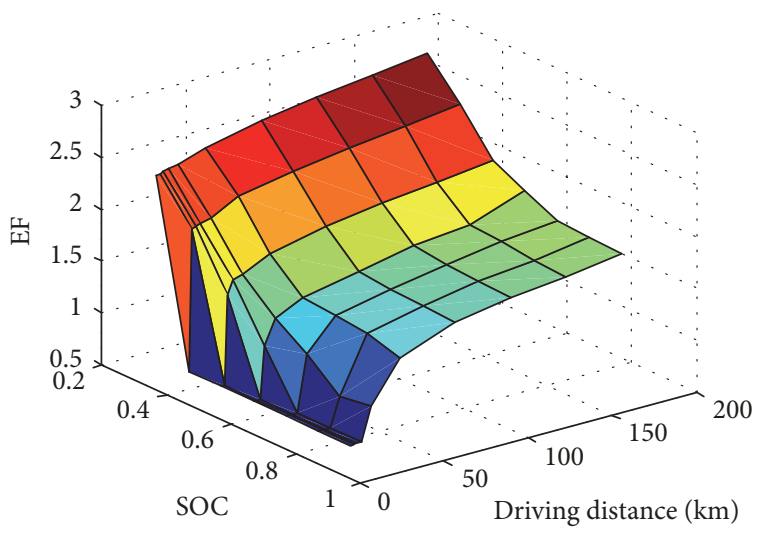

FIgURE 8: Optimal EF of high speed cycle.

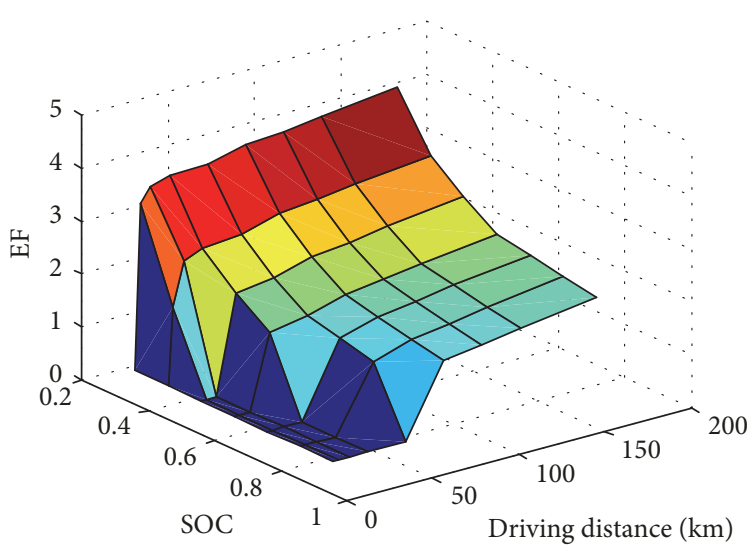

Figure 9: Optimal EF of middle speed cycle.

then these data will be used to identify the driving pattern according to the driving cycle recognition rules. It is noted that in the above recognition process, the initial driving pattern is set to the middle speed cycle.

In Step 6, the driving distance is got by navigation system and vehicle's velocity. The total distance can be obtained by navigation system. The completed distance can be calculated by the vehicle's velocity. Then driving distance is obtained by subtracting the completed distance from the total distance.

In Step 7, based on the aforementioned work, the EF can be obtained by looking up EF Maps through the type of driving cycle, driving distance, and SOC.

In Step 8, simplified-ECMS-based EMS is employed to solve the energy distribution optimization problem, and the optimal torque distribution between engine and motor can be obtained.

\section{Results and Discussions}

In this section, the proposed adaptive energy management strategy is verified in simulation. To evaluate the control performance of the proposed adaptive energy management strategy, the CD-CS-based strategy and the proposed strategy were simulated in MATLAB/Simulink, and the CD-CS-based strategy was taken as the benchmark.

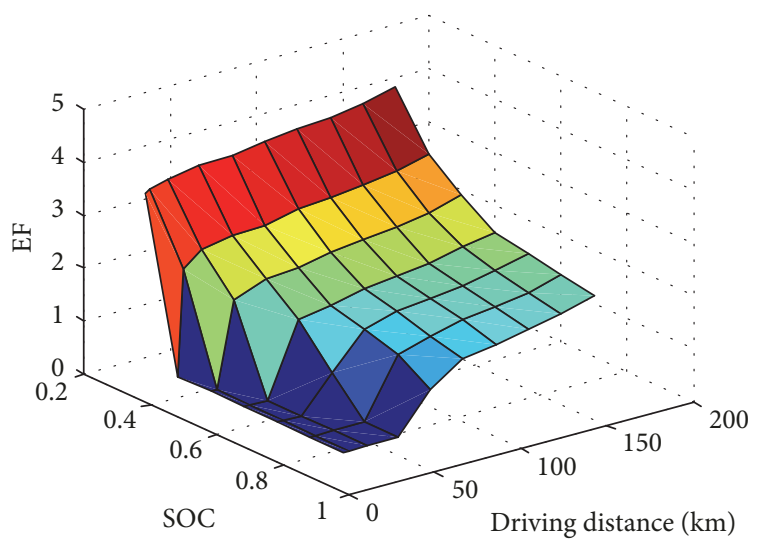

Figure 10: Optimal EF of low speed cycle.

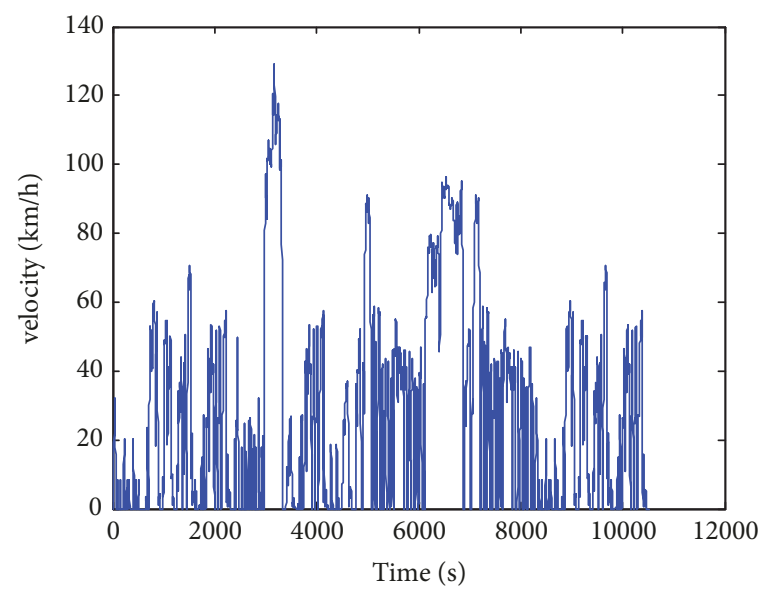

Figure 11: The test driving cycle.

TABLE 4: The calculation time of different control strategies under the test driving cycle.

\begin{tabular}{lc}
\hline & calculation time $^{*}(\mathrm{~s})$ \\
\hline CD-CS-based strategy & 72.23 \\
The proposed adaptive strategy & 164.39 \\
Simplified-ECMS-based strategy & 149.26 \\
ECMS-based strategy & 875.83 \\
\hline
\end{tabular}

${ }^{*}$ The calculation was completed on a laptop with 8 gigabits of RAM and 2.5 $\mathrm{GHz}$ of core i7 processor.

The simulation works are carried out under a test driving cycle. The driving cycle is shown in Figure 11. It is made of different representative driving cycles, such as NYGTC, CSHVR, UDDS, NewYorkBus, US06_HWY, WVUCITY, and HWFET. The speed profiles of these driving cycles are obtained from advanced vehicle simulator (Advisor), which is developed by the National Renewable Energy Laboratory of America. The simulation results are shown in Figures 12, 13, 14,15 , and 16. And the comparison of calculation time under different strategies is shown in Table 4.

The driving pattern recognition result is shown in Figure 12. On the $y$-axis, 1 stands for low speed cycle, 2 stands 


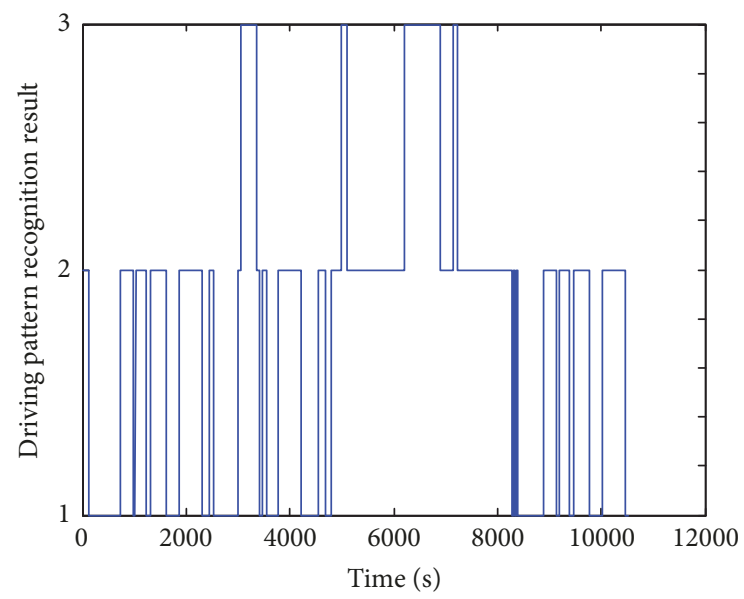

FIgURE 12: The driving pattern recognition result.
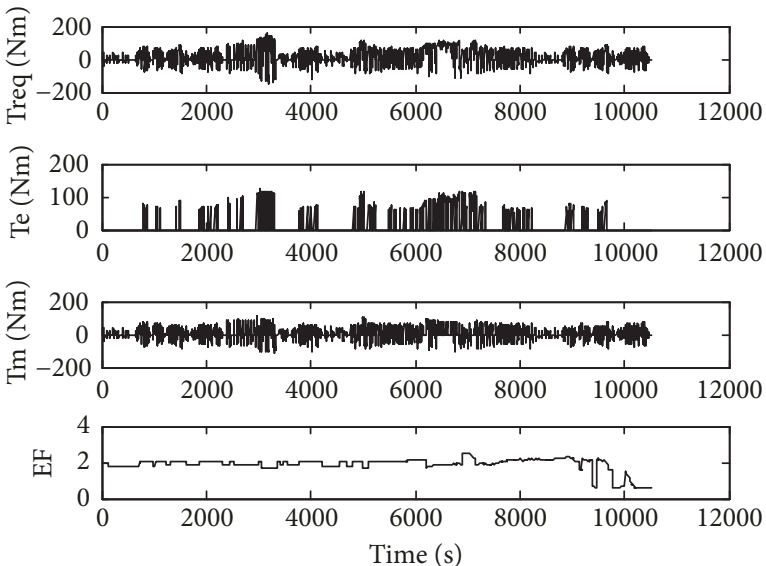

FIGURE 13: The basic results of the proposed strategy on the test driving cycle.

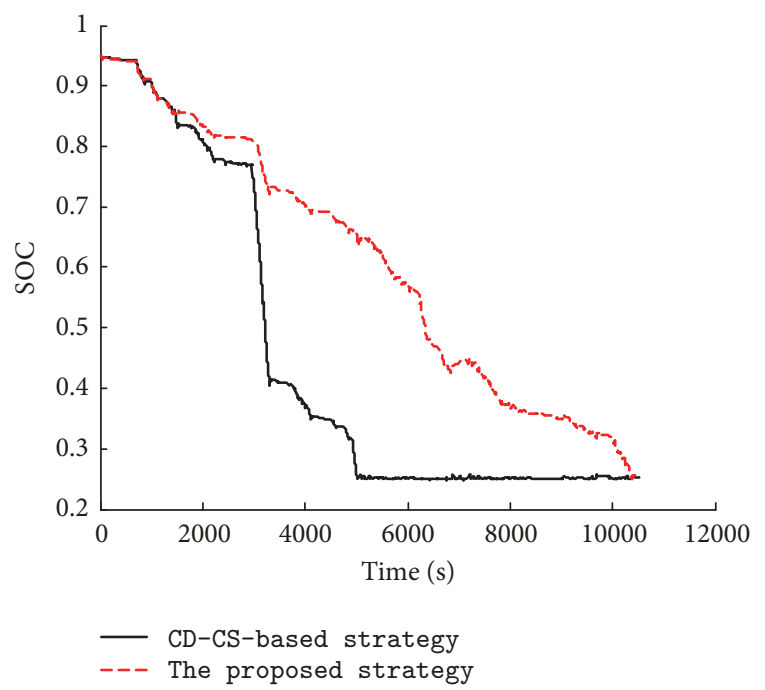

FIGURE 14: SOC variation trajectories.

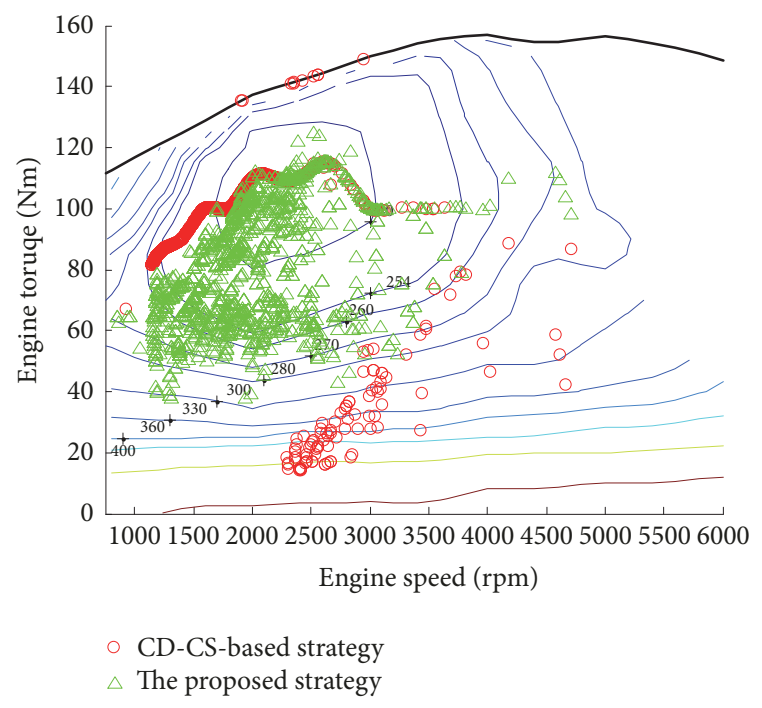

FIGURE 15: Engine working points on the test driving cycle.

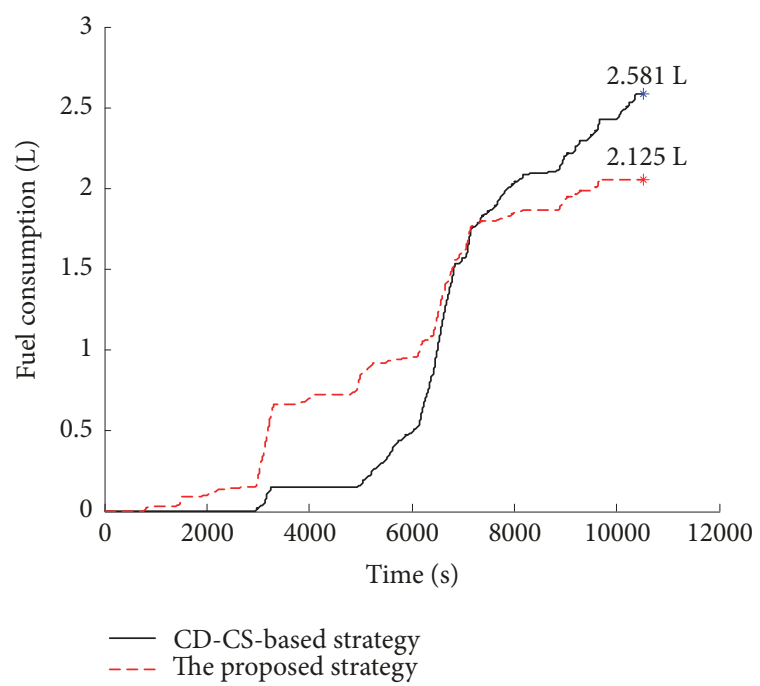

Figure 16: Fuel consumption curves on the test driving cycle.

for middle speed cycle, and 3 stands for high speed cycle. The results indicate that the driving cycle recognition algorithm in Step 5 of Section 4 can accurately and effectively identify the driving pattern.

The basic results, including the required torque of plugin hybrid powertrain $\left(T_{\text {req }}\right)$, engine torque $\left(T_{e}\right)$, motor torque $\left(T_{m}\right)$, and EF, are shown in Figure 13. The output torque of engine and motor could satisfy the required torque of plug-in hybrid powertrain to ensure the drivability of plug-in hybrid electric vehicle. The results of EF reflect that the proposed adaptive real-time strategy can adjust the $\mathrm{EF}$ according to the type of cycle, driving distance, and SOC, so the proposed adaptive real-time strategy executed the reasonable torque distribution combined with the optimized EF.

SOC curves of these two strategies on the test driving cycle were shown in Figure 14. As shown in this figure, the driving cycle has a relatively long distance and the battery 
energy alone is not enough to cover the entire driving cycle, so the battery of these two strategies are almost depleted. And the SOC under the CD-CS-based strategy decreased faster than the proposed adaptive strategy.

Engine working points of these two strategies on the test driving cycle are shown in Figure 15. As shown in this figure, most of the engine's working points under the proposed adaptive real-time strategy are distributed in the engine's economic zone. While lots of engine's working points under CD-CS-based strategy are away from this zone, the proposed adaptive strategy can effectively improve the engine's operation efficiency.

Fuel consumption curves of these two strategies on the test driving cycle are shown in Figure 16. From the figure, the fuel consumption under the CD-CS-based strategy is $2.581 \mathrm{~L}$. The fuel consumption under the proposed adaptive strategy is $2.125 \mathrm{~L}$; this strategy helps to reduce the fuel consumption significantly. Compared to CD-CS-based strategy, the proposed adaptive strategy reduces the fuel consumption by $17.63 \%$ under the testing driving cycle. Therefore, the proposed adaptive strategy has a good fuel economy performance.

The calculation time of different control strategies under the test driving cycle is shown in Table 4. The calculation time of the proposed adaptive strategy is slightly longer than the time of Simplified-ECMS-based strategy. The calculation time of the above two strategies is obviously shorter than the time of ECMS-based strategy, and they are close to the time of CD-CS-based strategy, which is a real-time control strategy. Therefore, we can conclude that it is very likely to apply the proposed adaptive energy management strategy for real-time control.

\section{Conclusions}

In this paper, an adaptive real-time energy management strategy based on simplified-ECMS and a novel driving pattern recognition method for PHEV is proposed. The findings of this paper can be shown as follows:

(1) A novel driving pattern recognition method based on statistical analysis has been proposed. This method classifies driving cycles into three driving patterns: low speed cycle, middle speed cycle, and high speed cycle, and then statistical analysis on these three driving patterns is carried to obtain rules. Simulation results show that the types of real-time driving cycles can be effectively identified according to these rules.

(2) Particle swarm optimization (PSO) algorithm is applied to optimize equivalent factor (EF) and then the EF MAPs, indexed vertically by battery's SOC and horizontally by driving distance, under the above three driving cycles are obtained.

(4) An adaptive real-time energy management strategy based on Simplified-ECMS and a novel driving pattern recognition has been proposed. Simulation on a test driving cycle is performed. The simulation results show that the adaptive energy management strategy can decrease fuel consumption of PHEV by $17.63 \%$ under the testing driving cycle, compared to CD-CS-based strategy. The calculation time of the proposed adaptive strategies is obviously shorter than the time of ECMS-based strategy and close to the time of CD-CS-based strategy, which is a real-time control strategy.

Currently, the proposed adaptive strategy is only verified through simulations. The next step is to perform hardwarein-the-loop test or experimental validations.

\section{Data Availability}

The data used to support the findings of this study are available from the corresponding author upon request.

\section{Conflicts of Interest}

The authors declare that they have no competing interests.

\section{Acknowledgments}

The work presented in this paper is supported by the National Natural Science Foundation of China (Grant no. 51665020) and the State Key Laboratory of Mechanical Transmission's open fund (Grant no. SKLMT-KFKT-201617).

\section{References}

[1] A. Gao, X. Deng, M. Zhang, and Z. Fu, "Design and Validation of Real-Time Optimal Control with ECMS to Minimize Energy Consumption for Parallel Hybrid Electric Vehicles," Mathematical Problems in Engineering, vol. 2017, pp. 1-13, 2017.

[2] X. Hu, C. M. Martinez, and Y. Yang, "Charging, power management, and battery degradation mitigation in plug-in hybrid electric vehicles: a unified cost-optimal approach," Mechanical Systems and Signal Processing, 2016.

[3] X. Zhou, D. Qin, and J. Hu, "Multi-objective optimization design and performance evaluation for plug-in hybrid electric vehicle powertrains," Applied Energy, vol. 208, pp. 1608-1625, 2017.

[4] Z. Lei, D. Cheng, Y. Liu, D. Qin, Y. Zhang, and Q. Xie, "A Dynamic Control Strategy for Hybrid Electric Vehicles Based on Parameter Optimization for Multiple Driving Cycles and Driving Pattern Recognition," Energies, vol. 10, no. 1, pp. 54-74, 2017.

[5] F. R. Salmasi, "Control strategies for hybrid electric vehicles: evolution, classification, comparison, and future trends," IEEE Transactions on Vehicular Technology, vol. 56, no. 5, pp. 23932404, 2007.

[6] Q. Gong, Y. Li, and Z. Peng, "Computationally Efficient Optimal Power Management for Plug-In Hybrid Electric Vehicles With Spatial Domain Dynamic Programming," in Proceedings of the ASME 2008 Dynamic Systems and Control Conference, pp. 10191026, Ann Arbor, Michigan, USA.

[7] X. Wu, X. Hu, X. Yin, and S. J. Moura, "Stochastic Optimal Energy Management of Smart Home With PEV Energy Storage," IEEE Transactions on Smart Grid, vol. 9, no. 3, pp. 20652075, 2018.

[8] X. Wang, H. He, F. Sun, and J. Zhang, "Application study on the dynamic programming algorithm for energy management of plug-in hybrid electric vehicles," Energies, vol. 8, no. 4, pp. 3225-3244, 2015.

[9] Z. Chen, C. C. Mi, B. Xia, and C. You, "Energy management of power-split plug-in hybrid electric vehicles based on simulated 
annealing and Pontryagin's minimum principle," Journal of Power Sources, vol. 272, pp. 160-168, 2014.

[10] Z. Chen, C. C. Mi, R. Xiong, J. Xu, and C. You, "Energy management of a power-split plug-in hybrid electric vehicle based on genetic algorithm and quadratic programming," Journal of Power Sources, vol. 248, pp. 416-426, 2014.

[11] M. Ansarey, M. Shariat Panahi, H. Ziarati, and M. Mahjoob, "Optimal energy management in a dual-storage fuel-cell hybrid vehicle using multi-dimensional dynamic programming," Journal of Power Sources, vol. 250, pp. 359-371, 2014.

[12] C. Zhang, A. Vahidi, P. Pisu, X. Li, and K. Tennant, "Role of terrain preview in energy management of hybrid electric vehicles," IEEE Transactions on Vehicular Technology, vol. 59, no. 3, pp. 1139-1147, 2010.

[13] D. Kum, H. Peng, and N. K. Bucknor, “Optimal energy and catalyst temperature management of plug-in hybrid electric vehicles for minimum fuel consumption and tail-pipe emissions," IEEE Transactions on Control Systems Technology, vol. 21, no. 1, pp. 14-26, 2013.

[14] S. G. Wirasingha and A. Emadi, "Classification and review of control strategies for plug-in hybrid electric vehicles," IEEE Transactions on Vehicular Technology, vol. 60, no. 1, pp. 111-122, 2011.

[15] C. M. Martinez, X. Hu, D. Cao, E. Velenis, B. Gao, and M. Wellers, "Energy Management in Plug-in Hybrid Electric Vehicles: Recent Progress and a Connected Vehicles Perspective," IEEE Transactions on Vehicular Technology, vol. 66, no. 6, pp. 4534-4549, 2017.

[16] M. Zhang, Y. Yang, and C. C. Mi, "Analytical approach for the power management of blended-mode plug-in hybrid electric vehicles," IEEE Transactions on Vehicular Technology, vol. 61, no. 4, pp. 1554-1566, 2012.

[17] X. Hu, N. Murgovski, L. Johannesson, and B. Egardt, "Energy efficiency analysis of a series plug-in hybrid electric bus with different energy management strategies and battery sizes," Applied Energy, vol. 111, pp. 1001-1009, 2013.

[18] R. Beck, A. Bollig, and D. Abel, "Comparison of two real-time predictive strategies for the optimal energy management of a hybrid electric vehicle," Oil \& Gas Science and Technology Revue d'IFP Energies nouvelles, vol. 62, no. 4, pp. 635-643, 2007.

[19] L. Xu, M. Ouyang, J. Li, F. Yang, L. Lu, and J. Hua, "Application of Pontryagin's minimal principle to the energy management strategy of plug-in fuel cell electric vehicles," International Journal of Hydrogen Energy, vol. 38, no. 24, pp. 10104-10115, 2013.

[20] S. Xie, H. Li, Z. Xin, T. Liu, and L. Wei, "A pontryagin minimum principle-based adaptive equivalent consumption minimum strategy for a plug-in hybrid electric bus on a fixed route," Energies, vol. 10, no. 9, pp. 1379-1399, 2017.

[21] Y. Zeng, Y. Cai, C. Chu, G. Kou, and W. Gao, "Integrated Energy and Catalyst Thermal Management for Plug-In Hybrid Electric Vehicles," Energies, vol. 11, no. 7, pp. 1761-1790, 2018.

[22] G. Paganelli, S. Delprat, and T. Guerra, "Equivalent consumption minimization strategy for parallel hybrid powertrain," in Proceedings of the IEEE 55th Vehicular Technology Conference (VTC '02), vol. 4, pp. 2076-2081, Birmingham, UK, May 2002.

[23] V. Sezer, M. Gokasan, and S. Bogosyan, "A novel ECMS and combined cost map approach for high-efficiency series hybrid electric vehicles," IEEE Transactions on Vehicular Technology, vol. 60, no. 8, pp. 3557-3570, 2011.

[24] J. Han, Y. Park, and D. Kum, "Optimal adaptation of equivalent factor of equivalent consumption minimization strategy for fuel cell hybrid electric vehicles under active state inequality constraints," Journal of Power Sources, vol. 267, pp. 491-502, 2014.

[25] Y. Gao and M. Ehsani, "Design and control methodology of plug-in hybrid electric vehicles," IEEE Transactions on Industrial Electronics, vol. 57, no. 2, pp. 633-640, 2010.

[26] B. Egardt, N. Murgovski, M. Pourabdollah et al., "Electromobility studies based on convex optimization: design and control issues regarding vehicle electrification," IEEE Control Systems Magazine, vol. 34, no. 2, pp. 32-49, 2014.

[27] C. Hou, M. Ouyang, L. Xu, and H. Wang, "Approximate Pontryagin's minimum principle applied to the energy management of plug-in hybrid electric vehicles," Applied Energy, vol. 115, pp. 174-189, 2014.

[28] C. Yang, S. Du, L. Li, S. You, Y. Yang, and Y. Zhao, "Adaptive realtime optimal energy management strategy based on equivalent factors optimization for plug-in hybrid electric vehicle," Applied Energy, vol. 203, pp. 883-896, 2017.

[29] A. Sciarretta, L. Serrao, and P. C. Dewangan, "A control benchmark on the energy management of a plug-in hybrid electric vehicle," Control Engineering Practice, vol. 29, pp. 287298, 2014.

[30] S. Stockar, V. Marano, M. Canova, G. Rizzoni, and L. Guzzella, "Energy-optimal control of plug-in hybrid electric vehicles for real-world driving cycles," IEEE Transactions on Vehicular Technology, vol. 60, no. 7, pp. 2949-2962, 2011.

[31] Y. Zeng, Y. Cai, G. Kou, W. Gao, and D. Qin, “Energy Management for Plug-In Hybrid Electric Vehicle Based on Adaptive Simplified-ECMS," Sustainability, vol. 10, no. 6, pp. 2060-2084, 2018 . 


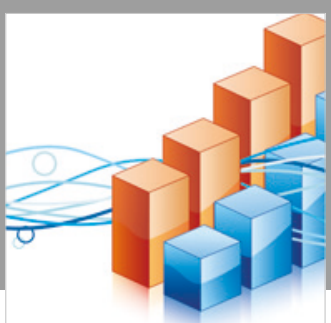

Advances in

Operations Research

\section{-n-m}
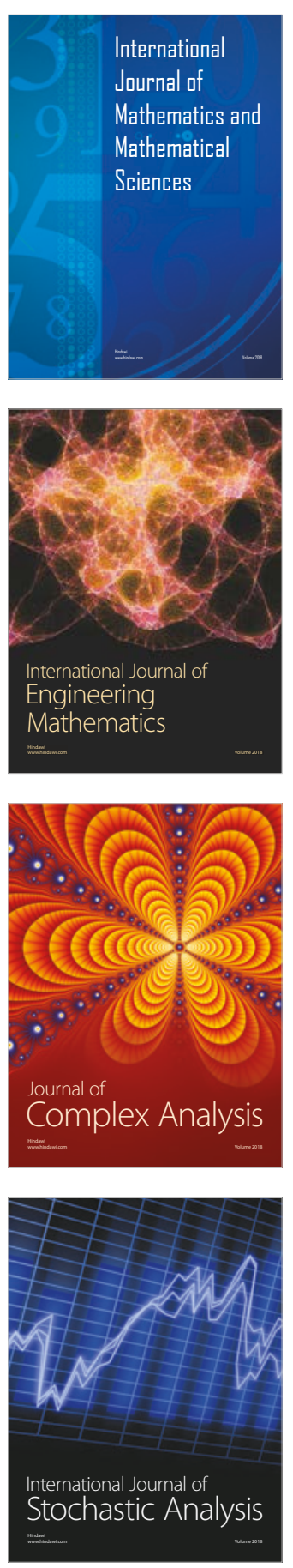
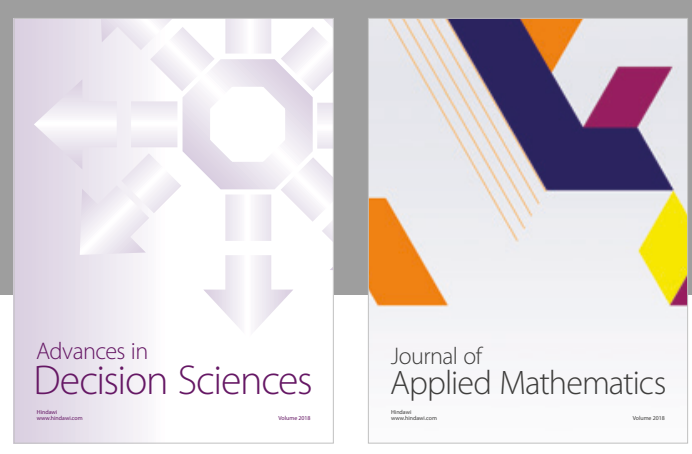

Journal of

Applied Mathematics
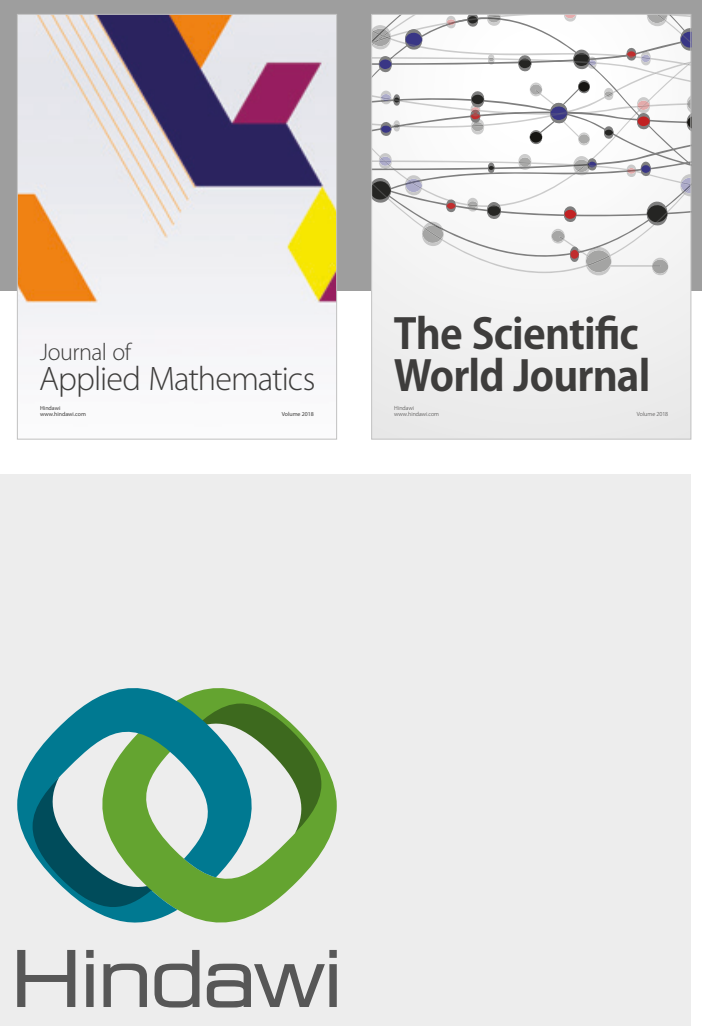

Submit your manuscripts at

www.hindawi.com

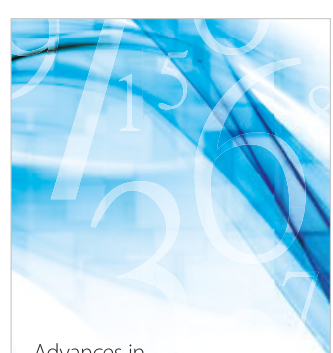

Advances in
Numerical Analysis
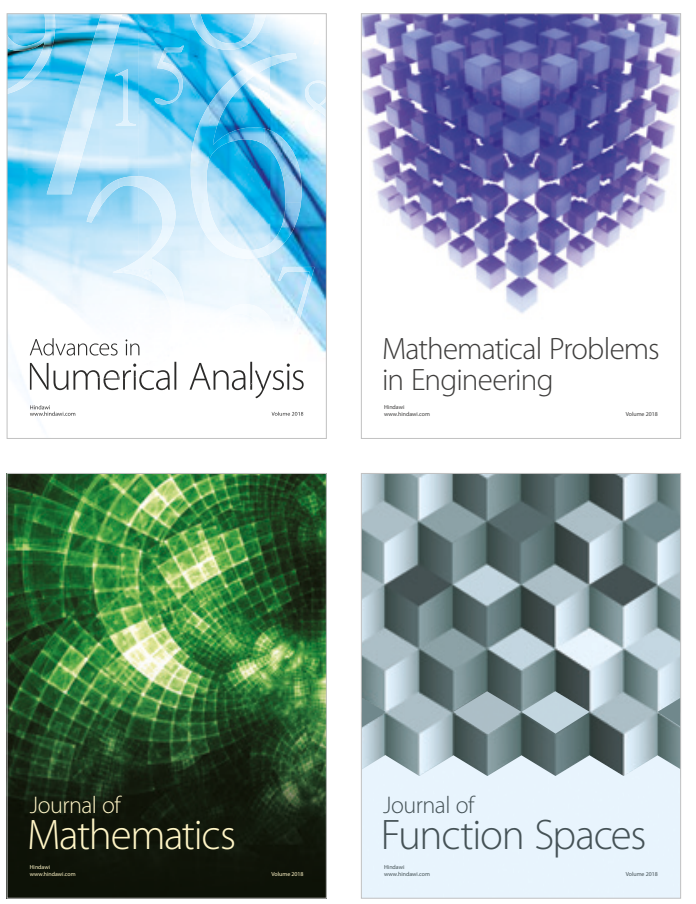

Mathematical Problems in Engineering

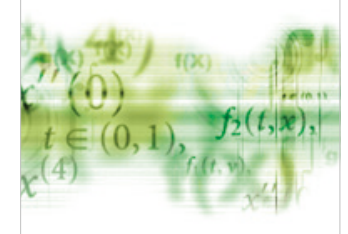

International Journal of

Differential Equations

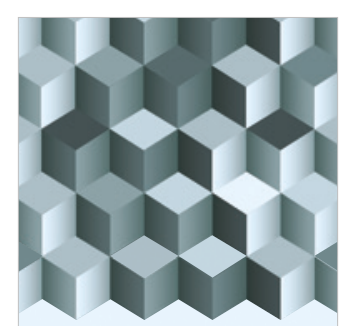

Journal of

Function Spaces

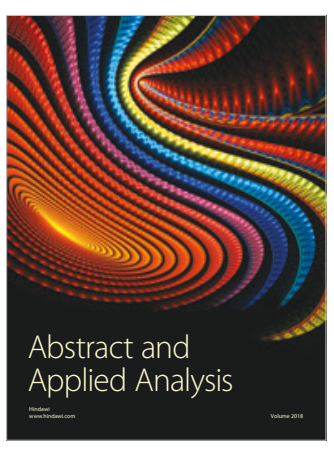

The Scientific

World Journal

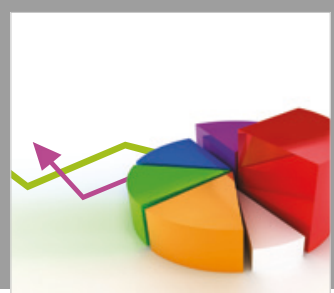

Journal of

Probability and Statistics
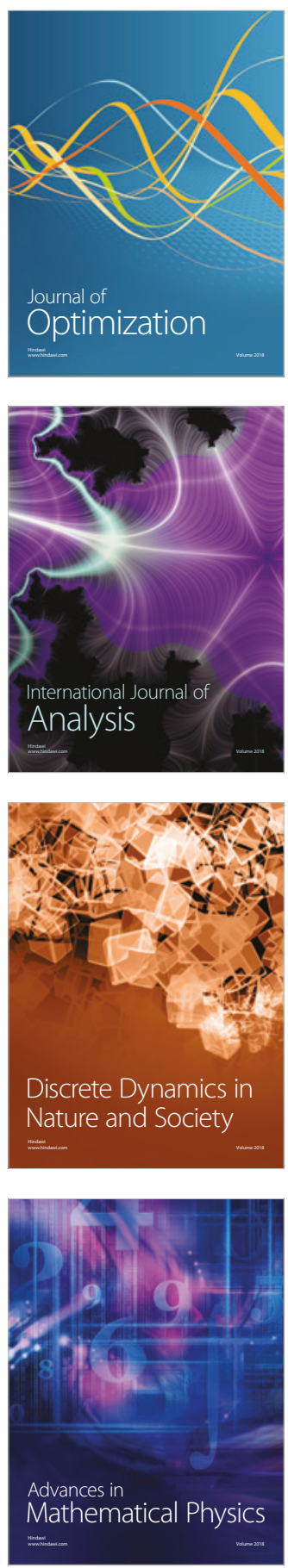